\title{
Validation of a Hybrid Electric Vehicle Dynamics Model for Energy Management and Vehicle Stability Control
}

\author{
K. Reeves \\ Motorsport Engineering \\ National Motorsport Academy, Nottingham, UK \\ kieran@motorsport.nda.ac.uk
}

\author{
A. Montazeri and C.J. Tayor \\ Engineering Department \\ Lancaster University, Lancaster, UK \\ a.montazeri@lancaster.ac.uk, c.taylor@lancaster.ac.uk
}

\begin{abstract}
A Simulink ${ }^{\circledR}$ Hybrid Electric Vehicle dynamics model for the control of energy management and vehicle stability is developed. The model encompasses a transitional vehicle speed input parameterized by the New European Driving Cycle. Internal combustion engine torque, motor torque and varying corner radii are set to the same time constraints as the drive cycle. Lateral acceleration, yaw rate and tyre data are validated against measured car data, resulting in a simulation model that can be utilised (with modifications) as a tool to determine stability control and power deployment for front-wheel, rearwheel or all-wheel drive hybrid vehicles. The model yields similar outputs to a driven vehicle's normal measured responses.
\end{abstract}

Keywords - Hybrid Electric Vehicles; Longitudinal Dynamics; Lateral Dynamics; Vehicle Dynamics; Energy Management; Stability Control; Drive Cycles.

\section{INTRODUCTION}

Simulation models are the focus for the majority of the automotive industry especially within the research and development sector. In Motorsport especially, Formula One and World Endurance Championship teams tend to use simulators that emulate the physical car, allowing various scenarios and set-ups to be considered and perfected long before they reach a racetrack. Although this paper uses the improved 'real-world' driving scenario of the new European driving cycle (NEDC), the simulation could also be utilised to determine correct steering and throttle/braking inputs of a driver to improve their lap times. The current European test for emissions data, the NEDC, comprises a start-stop type scenario and various speeds to resemble around town and motorway (freeway) driving. The drive cycle lasts for 1180 s over a distance of $11017 \mathrm{~m}$. The average speed of the test is $33.6 \mathrm{~km}-\mathrm{h}$ [1].

Current vehicle dynamics simulation platforms do not allow for closed-loop active control of vehicle stability. Simulation platforms such as Adams Car and GT-Drive require third party coupling to software such as Simulink ${ }^{\circledR}$ to enable control of the vehicle. Furthermore, usually pre-defined vehicle speed and cornering look-up table data are used as maxima for cornering forces when not coupled to externally produced systems [2,3]. Vehicle models are normally classed as one of two types: forward looking models and backward facing models [4].
Backward looking models make the assumption that the vehicle meets the target performance, and calculates the component states. Forward- facing models, on the other hand, simulate the physical behaviour of each component, include control instructions, handle state changes, and generate vehicle performance as outputs. Advanced controlled co-simulations are typically developed with, for example, fuzzy control [5]. Various authors have developed longitudinal only dynamic simulation models for the purpose of optimizing energy management strategies for emission drive cycle testing e.g. [6-8]. The 2016 US Volkswagen scandal [9] highlighted inaccuracies in manipulated vehicle data but also showed that, as many believed in the industry, one dimensional testing does not accurately produce real world driving emissions [10].

The present article describes the development of a combined longitudinal and lateral dynamics Simulink ${ }^{\circledR}$ model that will enable a control system to be amalgamated to include energy management strategies and stability control during a twodimensional (yaw plane) drive cycle derived from the NEDC. Equations of motion are developed for longitudinal motion, yaw rate and under/over steer displacement. These equations are combined and used as the initial vehicle platform to be controlled. The model is forward looking in that vehicle speed is controlled alongside steering input, allowing development of controllers for vehicle stability. Longitudinal vehicle speed with time, engine torque and road curvature are the sole inputs for the simulation, whilst lateral speed, yaw acceleration rate and its derivatives, and vehicle trajectory are calculated outputs.

The objective of the simulation is to calculate tractive effort and resistance forces to determine longitudinal net vehicle force at the road and vehicle trajectory during a turn. These results will be utilised as inputs for a future control system that will allow maximum tractive effort and appropriate energy regeneration to be determined, whilst maintaining neutral steer for the duration of the route. The paper is organized as follows. Section II reviews longitudinal acceleration dynamics, resistive forces, tractive forces and net work at the tyre-road surface. Section III describes the lateral model, a derivative of the elementary bicycle model, whilst section IV details the simulation model and validation results. Finally, the conclusions are summarised in section $\mathrm{V}$. 


\section{LONGITUDINAL DYNAMICS}

Tractive force is subject to its mass and acceleration. Vehicle acceleration is defined as follows:

$$
\dot{V}_{x}=\frac{F_{t}-F_{r}}{M}
$$

where $V_{x}$ is longitudinal vehicle speed, $M$ is the vehicle mass, $F_{t}$ is tractive effort (total) and $F_{r}$ is resistance (total), where the latter is given by $R_{r}+F_{g}+D_{A}$, in which $R_{r}$ is the rolling resistance, $F_{g}$ is the road grade resistance and $D_{A}$ the aerodynamic drag. Vehicle resistance is described as the total forces opposing the vehicle movement. This could be in the form of road gradient (uphill) resistance, aerodynamic drag and rolling resistance of the tyre at the road surface.

\section{A. Road Grading Resistance}

When a vehicle attempts to ascend an incline the mass of the vehicle creates a resistive force against the vehicle. The uphill grading resistance with a road angle $(\alpha)$ can be expressed,

$$
F_{g}=M g \sin \alpha
$$

where $g$ is the acceleration due to gravity $\left(9.81 \mathrm{~m} / \mathrm{s}^{2}\right)$. Hence, the associated element or 'block' of the Simulink ${ }^{\circledR}$ model developed later is defined as follows:

Input for road grade block: vehicle Mass, acceleration due to gravity and road slope angle. Output from block: road grade resistance force for rolling resistance equation.

\section{B. Rolling Resistance}

The rolling resistance of a vehicle is typically due to the tyre contact patch with the road and the hysteresis of the tyre compound and materials [11]. Fig. 1 shows the distribution of pressure at the contact patch when a vehicle is stationary, in which $P$ is the force acting at the centre of the wheel and $P_{z}$ is the reacting force, aligned with $P$, and the deformation is classified as $z$. When a vehicle travels on hard road surface, the tyre pressure distribution tends to deflect as shown in Fig. 1 (a) and the resultant reaction force relocates a distance $d$ from the centre of the wheel. The ground reaction to the intended forward motion is the rolling resistant moment:

$$
T_{r}=P d
$$

To maintain wheel rotation, the force acting on the centre of the wheel is required and must balance the rolling resistant moment. The rolling resistant moment can be substituted for a horizontal force that is acting on the centre of the wheel but in the opposite direction than the wheel is moving, otherwise known as rolling resistance:

$$
R_{r}=P r_{c}
$$

where $r_{c}$ is the rolling resistance co-efficient. This rolling resistance coefficient is a function of the tyre properties and environmental conditions, primarily tyre materials, structure, tread pattern, tyre pressure, temperature, road material and the

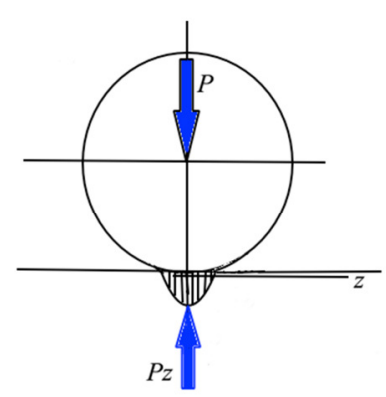

\begin{tabular}{|c|c|}
\hline \multicolumn{2}{|c|}{ Various Road Conditions vs Rolling Resistance [12] } \\
\hline Condition & $r_{c}$ \\
\hline Car tyres on a concrete or asphalt road & 0.013 \\
\hline Car tyres on a rolled gravel road & 0.02 \\
\hline Tar macadam road & 0.025 \\
\hline Unpaved road & 0.05 \\
\hline Field & $0.1-0.35$ \\
\hline Wheel on iron rail & $0.001-0.002$ \\
\hline
\end{tabular}

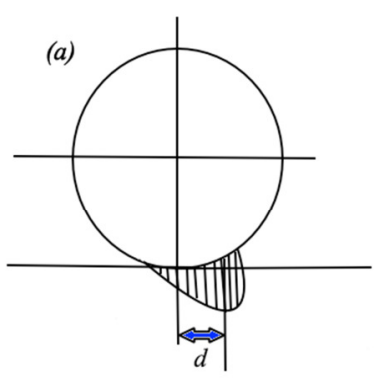

Figure 1. Tyre pressure distribution.

TABLE I. ROLLING RESISTANCE.

road adhesion qualities, for example the presence of rain or spilled liquids. Table I shows typical coefficients for varying road types. For typical vehicle dynamics calculations it is sufficient to assume resistance is a linear function of speed. The Bosch Handbook [13] states that for a common vehicle with tyre pressures in a normal range, on concrete, travelling up to speeds of $128 \mathrm{~km} / \mathrm{h}$, the rolling resistance coefficient can be expressed as:

$$
r_{c}=0.01\left(1+\frac{V_{x}}{160}\right)
$$

The rolling resistance and grading resistance can be combined as road resistance:

$$
M g\left(r_{c} \cos \alpha+\sin \alpha\right)
$$

Hence, the associated element of the Simulink $\AA$ model is:

Input for rolling resistance block: road grade resistive force, vehicle speed. Output from block: road grade and rolling resistance force for total resistive forces equation.

\section{Aerodynamic Resistance}

Drag is a function of air density, the vehicle body shapes coefficient of drag, the frontal area and the vehicle speed. Aerodynamic drag as defined in equation (7) resists the vehicle forward motion and increases with speed:

$$
D_{A}=0.5 \rho A_{f} C_{D} V_{x}^{2}
$$

where $\rho$ is the air density, $A_{f}$ the vehicle's frontal area and $C_{D}$ the body's coefficient of drag. The headwind speed can also influence the aerodynamic drag and can be accounted for but is not included in this model. Hence: 
Input for aerodynamic resistance block: environment (air density), vehicle parameters from script (frontal area, drag coefficient) and vehicle speed. Output from block: drag resistive force for total resistive force block.

\section{Longitudinal Equations of Motion}

When a vehicle is moving, the major forces applied externally to the vehicle are the rolling resistance of both the front and rear tyres, expressed as rolling resistance moment (3), up-hill climbing resistance (2), tractive effort of both front $\left(F_{t f}\right)$ and rear wheels $\left(F_{t r}\right)$ (zero for a non-driven axle) and aerodynamic drag (7). The vehicle motion in this longitudinal direction can be expressed with a dynamic equation (8) derived from (1). Here, $R_{r f}$ and $R_{r r}$ are the rolling resistance of the front and rear tyres respectively.

$$
M \frac{V_{x}}{t}=\left(F_{t f}+F_{t r}\right)-\left(R_{r f}+R_{r r}+D_{A}+F_{g}\right)
$$

Equation (8) yields the linear acceleration over a distance and the mass of the vehicle. The equation is based on the sum of the resistive forces subtracted from the vehicles total tractive effort. To determine tractive effort, the normal load on the vehicle axles needs to be calculated. The sum of all moments of forces about the centre point of the tyre and ground can be used to determine the normal load on the front axle (9) and rear axle (10). A typical passenger car centre height of aerodynamic resistance, $h_{w}$, is assumed to be near the height of the centre of gravity ( $\mathrm{CoG})$ of the vehicle $\left(h_{g}\right)$ [12]. The load is determined by the wheelbase $(L)$, the distance from the front and rear axles to the $\mathrm{CoG}, L_{a}$ and $L_{b}$ respectively, and finally the radius of the tyre $r_{d}$. Utilising equations (4) and (8),

$$
\begin{aligned}
& W_{f}=\frac{L_{b}}{L} M g \cos \alpha-\frac{h_{g}}{L}\left(F_{t}-R_{r}\left(1-\frac{r_{d}}{h_{g}}\right)\right) \\
& W_{r}=\frac{L_{a}}{L} M g \cos \alpha-\frac{h_{g}}{L}\left(F_{t}-R_{r}\left(1-\frac{r_{d}}{h_{g}}\right)\right)
\end{aligned}
$$

The tyre to ground contact patch can only support up to a maximum value. Even a small amount over the maximum and tractive effort will cause the tyre to lose traction and the tyre will spin. This maximum is the frictional coefficient and is a product of the coefficient of adhesion on the road $(\mu)$ and the normal load. Front and rear maximum tractive effort, $F_{m f}$ and $F_{m r}$ are expressed as follows:

$$
\begin{gathered}
F_{m f}=\frac{\mu_{i} M g \cos \alpha\left[L_{b}+f_{r}\left(h_{g}-r_{d}\right)\right] / L}{1+\mu_{i} h_{g} / L} \\
F_{m r}=\frac{\mu_{i} M g \cos \alpha\left[L_{a}+f_{r}\left(h_{g}-r_{d}\right)\right] / L}{1+\mu_{i} h_{g} / L}
\end{gathered}
$$

TABLE II. ROAD SURFACE CO-EFFICIENT [12].

\begin{tabular}{|l|l|l|}
\hline \multicolumn{3}{|c|}{ Average Values of Tractive Effort Coefficient on Various Roads } \\
\hline Surface & Peaking Values $\boldsymbol{\mu}_{\boldsymbol{\rho}}$ & Slipping Values $\boldsymbol{\mu}_{\mathrm{s}}$ \\
\hline $\begin{array}{l}\text { Asphalt and concrete } \\
\text { (dry) }\end{array}$ & $0.8-0.9$ & 0.75 \\
\hline Concrete (wet) & 0.8 & 0.7 \\
\hline Asphalt (wet) & $0.5-0.7$ & $0.45-0.6$ \\
\hline Gravel & 0.6 & 0.55 \\
\hline Earth road (dry) & 0.68 & 0.65 \\
\hline Earth road (wet) & 0.55 & $0.4-0.5$ \\
\hline Snow (hard packed) & 0.2 & 0.15 \\
\hline Ice & 0.1 & 0.07 \\
\hline
\end{tabular}

Here, $\mu_{i}$ is given by $\mu_{p}$ for acceleration and $\mu_{s}$ for braking as shown in Table II. At any given moment the maximum torque supplied from the internal combustion engine or an electric motor through the relevant transmission and drive-train components to the wheel should not exceed the tyre to ground cohesion or wheel spin will occur. The average tractive effort for various road surface materials is shown in Table II. Equations (11) and (12) are both utilised in the case of an AllWheel-Drive vehicle, while either are dismissed or set to zero in front-wheel or rear-wheel drive scenarios. The associated element of the Simulink® model is defined as follows:

Input for longitudinal dynamics: sum of total resistance (drag, rolling resistance, grading resistance), tyre parameters (rim diameter, tyre width and tyre aspect ratio of side wall), vehicle parameters (mass, wheelbase, weight distribution to ascertain CoG location and front and rear wheelbase lengths), gear ratio, engine/motor torque, vehicle speed, surface co-efficient and drive cycle time. Output for results or to be used as a basis to develop Hybrid Electric drivetrain architecture and energy management strategies (section IV): total resistive force, total tractive effort (front/rear).

\section{LATERAL DYNAMICS}

For this article, initial modeling and development of the equations of motion for lateral dynamics will be formed using an augmented version of the two degrees of freedom (2DOF) elementary automobile, usually described as the bicycle model, as illustrated in Fig. 2. The elementary 'bicycle' model is based on the following assumptions:

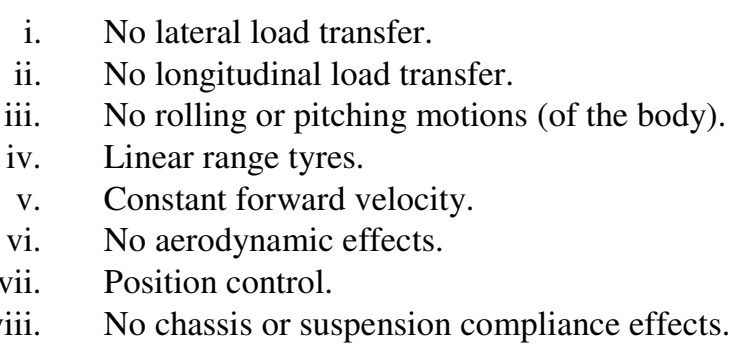

The simulation used here differs from the elementary model in that a dynamic forward velocity (third degree of freedom) is utilised, as determined by the drive cycle, and the following Pacejka tyre model (13) is used to determine tyre performance range [14]: 


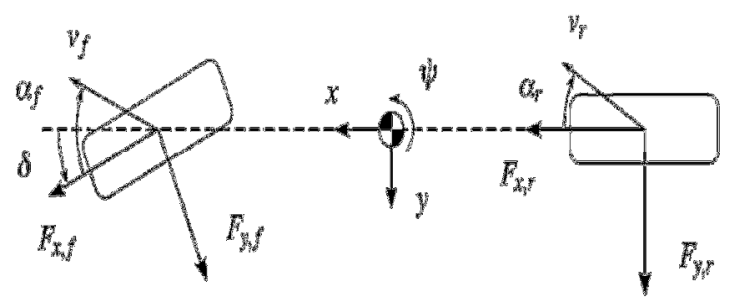

Figure 2. Bicycle Model Nomenclature [15].

$$
\begin{gathered}
F_{y, i}=\mu_{y, i} F_{z, i} \sin \left(C _ { y , i } \operatorname { a r c t a n } \left(B_{y, i} \alpha_{i}-E_{y, i}\left(B_{y, i} \alpha_{i}-\right.\right.\right. \\
\left.\left.\left.\arctan B_{y, i} \alpha_{i}\right)\right)\right)
\end{gathered}
$$

where $i$ determines the front or rear tyre, $\mu_{y}$ is the lateral friction co-efficient, $C_{y, i}$ is the shaping factor and $E_{y, i}$ determines the curvature. The stiffness factor is expressed as:

$$
B_{y, i}=\frac{C_{\alpha, i}}{C_{y, i} \mu_{y, i} F_{z, i}}
$$

Whilst vertical front and rear load can be determined by:

$$
F_{z, f}=M g \frac{L_{b}}{L} \quad F_{z, r}=M g \frac{L_{a}}{L}
$$

The slip angle $\left(\alpha_{i}\right)$ is determined by:

$$
\begin{gathered}
\alpha_{f}=\delta-\arctan \left(\frac{v_{y}+L_{a} \dot{\psi}}{V_{x}}\right) \\
\alpha_{r}=-\arctan \left(\frac{v_{y}+L_{b} \dot{\psi}}{v_{x}}\right)
\end{gathered}
$$

In this model, the input variables are the steered front wheel angle $(\delta)$ and longitudinal vehicle speed $\left(V_{x}\right)$. The two degrees of freedom referred to are the motion variables, i.e. yaw velocity $(\dot{\psi})$ and lateral velocity $\left(v_{y}\right)$. Positive yaw movement is in a clockwise direction and positive lateral movement is toward the right side of the vehicle. When the driver enters a corner a steer wheel angle $\left(\delta_{\mathrm{sw}}\right)$ is applied to negotiate the turn, and the wheel angle $(\delta)$ will be a ratio of this angle determined by the steering rack gearing. The vehicle at this stage would normally experience three stages to successfully negotiate the turn: transient turn entry, steady state and transient turn exit. From Newton's second law, the differential equations for torque and force are developed. In the case of the vehicle lateral dynamics model, for yawing moment $(\mathrm{N})$ and force in the $\mathrm{y}$-axis $(\mathrm{Y})$ :

$$
\begin{aligned}
& N=I_{z} \dot{\psi} \\
& Y=M a_{y}
\end{aligned}
$$

The elementary bicycle model in Fig 2, derived from equations (18) and (19) is determined by:

$$
M v_{y}+M V_{x} \dot{\psi}=F_{y, f}+F_{y, r}
$$

$$
I_{z} \ddot{\psi}=F_{y, f} L_{a}-F_{y, r} L_{b}
$$

where $I_{z}$ is the vehicle's inertia about the $z$ axis. With the inclusion of longitudinal dynamics $\left(F_{x}\right)$ and variable steering, both (20) and (21) can be rearranged to make $\ddot{\psi}$ and $v_{y}$ the product, hence the following simultaneous equations can be utilised within the Matlab®/Simulink® model:

$$
\begin{gathered}
\ddot{\psi}=\frac{L_{a} F_{y, f} \cos \delta-F_{y, r} L_{b}+L_{a} F_{x, f} \sin \delta}{I_{z}} \\
\dot{v}_{y}=\frac{F_{y, f} \cos \delta+F_{y, r}+F_{x} \sin \delta-M V_{x} \dot{\psi}}{M}
\end{gathered}
$$

Inputs and parameters for lateral dynamics: steered wheel angle (rad), vehicle speed, front and rear wheelbase, vehicle inertia, vehicle mass, acceleration due to gravity, Pacejka coefficients and tyre cornering stiffness. Outputs for stability control: yaw acceleration, velocity and displacement, lateral acceleration, velocity and displacement, front and rear tyre force.

\section{MATLAB® SIMULINK® IMPLEMENTATION}

\section{A. Model Validation}

Longitudinal simulation results can be validated against typical drive cycle simulations. However, as the input torque was supplied from a GT-Drive (backward looking model) simulation, in this instance simple hand calculation at any given speed will suffice for such a model. In the event of creating a backwards looking model for energy management control, the desired wheel force is incorporated into the model and resistive forces added via equation (24) to determine engine/motor torque requirements. The output is fed through the wheel/tyre and transmission multipliers.

$$
F_{\text {reqtyre }}=M \frac{d V_{x}}{d t}+g M \sin \alpha+g \cos \alpha M\left(f_{1}+\frac{V_{x}}{f_{2}}\right)+D_{A}
$$

where $f_{1}$ is the inflation tire pressure offset and $f_{2}$ is the inflation tire pressure coefficient.

Lateral simulation results were compared to measured data from vehicle tests carried out at Linköping University, Sweden [16] and from measured racecar logged data during a lap of the Brands Hatch Circuit, Kent, UK. At the Linköping University, a Volkswagen Golf had been utilised to carry out the double lane change (DLC) maneuver [17] over three steady state speeds, whilst instrumentation fitted to the car and original sensors measured through the ECU were monitored and recorded to provide accurate data. The steering data from these tests, illustrated in Fig. 3, and the vehicle specifications, stated in Table III, were utilised as an input for the Simulink® model and the simulation output responses were compared with the measured outputs. 
TABLE III. VOLKSWAGEN GOLF DATA

\begin{tabular}{|c|c|c|}
\hline Nomenclature & Unit & Value \\
\hline $\mathbf{m}$ & $\mathrm{kg}$ & 1415 \\
\hline $\mathbf{L}_{\mathbf{a}}$ & $\mathrm{m}$ & 1.03 \\
\hline $\mathbf{L}_{\mathbf{b}}$ & $\mathrm{m}$ & 1.55 \\
\hline $\mathbf{V}_{\mathbf{x}}$ & $\mathrm{km} / \mathrm{h}$ & $38 / 51 / 62$ \\
\hline $\mathbf{I}_{\mathbf{z}}$ & $\mathrm{kgm}$ & 2581 \\
\hline $\mathbf{C}_{\boldsymbol{\alpha}, \mathbf{f}}$ & $\mathrm{kN} / \mathrm{rad}$ & 103.6 \\
\hline $\mathbf{C}_{\boldsymbol{\alpha}, \mathbf{r}}$ & $\mathrm{m} / \mathrm{s}^{2}$ & 120 \\
\hline $\mathrm{g}$ & Dimensionless & 9.81 \\
\hline $\mathbf{C}_{\mathbf{y}, \mathbf{f}}$ & Dimensionless & 1.15 \\
\hline $\mathbf{C}_{\mathbf{y}, \mathbf{r}}$ & Dimensionless & 1.46 \\
\hline $\boldsymbol{\mu}_{\mathbf{y}, \mathbf{f}}$ & Dimensionless & 1.2 \\
\hline $\boldsymbol{\mu}_{\mathbf{y}, \mathbf{r}}$ & Dimensionless & 0.85 \\
\hline $\mathbf{E}_{\mathbf{y}, \mathbf{f}}$ & Dimensionless & 0.41 \\
\hline $\mathbf{E}_{\mathbf{y}, \mathbf{r}}$ & radians & -1.55 \\
\hline $\boldsymbol{\delta}$ & & See Fig 3 \\
\hline
\end{tabular}

Utilising the Pacejka magic formula requires good tyre input solutions acquired from tyre testing for a particular brand and compound. In this instance, tyre data was taken from Lundahl et al. [16] where 23 DLC maneuvers were carried out and a standard deviation determined: Table IV. From these data, a sensitivity response analysis for high standard deviation results is carried out to minimise the error. An initial run utilising standard tyre data yields results that interacted with appropriate responses in the time domain. However, peak values were high for lateral acceleration and low for yaw rate. The former was due to the vehicle speed from the measured vehicle data not staying constant throughout the manoeuvre, hence utilising a transient speed input for the simulation rectified this issue somewhat to within a $6 \%$ error. Changing the cornering stiffness of the front tyre within the standard deviation threshold ensured the yaw rate response was close to the measured data, as shown in Fig. 4.

Lateral velocity was initially faster for the simulated model, but again manipulation of the input data, in this case tyre data and inertia around the $z$-axis, changed the response rate. To support the model validation, a secondary test was conducted utilising data taken from a Lotus Evora GTE during a GT-Cup event. The data were taken from the steering input and the vehicle speed sensors were once again used as an input. Tyre data were manipulated to coincide with the Pirelli tyres used. Mass, wheelbase and centre of gravity location was measured using corner weight scales.

TABLE IV. VOLKSWAGEN GOLF DATA.

\begin{tabular}{|c|c|c|}
\hline Nomenclature & Value & $\begin{array}{c}\text { Standard } \\
\text { Deviation }\end{array}$ \\
\hline $\boldsymbol{C}_{\boldsymbol{\alpha}, \boldsymbol{f}}$ & $103600 \mathrm{~N} / \mathrm{rad}$ & 701 \\
\hline $\boldsymbol{C}_{\boldsymbol{\alpha}, \boldsymbol{r}}$ & $120000 \mathrm{~N} / \mathrm{rad}$ & 1288 \\
\hline $\boldsymbol{C}_{\boldsymbol{y}, \boldsymbol{f}}$ & 1.15 & 0.86 \\
\hline $\boldsymbol{C}_{\boldsymbol{y}, \boldsymbol{r}}$ & 1.46 & 0.055 \\
\hline $\boldsymbol{\mu}_{\boldsymbol{y} \boldsymbol{f}}$ & 1.2 & 0.079 \\
\hline $\boldsymbol{\mu}_{\boldsymbol{y}, \boldsymbol{r}}$ & 0.85 & 0.002 \\
\hline $\boldsymbol{E}_{\boldsymbol{y}, \boldsymbol{f}}$ & 0.41 & 2.18 \\
\hline $\boldsymbol{E}_{\boldsymbol{y}, \boldsymbol{r}}$ & -1.55 & 0.19 \\
\hline
\end{tabular}

Finally, lateral acceleration data were taken from the Lotus' G-sensor and compared with the Simulink model, as illustrated by Fig 5. Once again the data were within reasonable error constraints taking into account tyre properties are not exact and the roll and pitch of the vehicle is ignored.

\section{B. Longitudinal and Lateral Simulation}

Once accurate responses had been achieved the combined longitudinal and lateral model could be created. Using the NEDC and an appropriate engine/motor torque profile as the model inputs, a longitudinal model is implemented to determine vehicle tractive effort, axle work and vehicle resistance. Utilising equations (6) and (7), aerodynamic drag, road gradient (for this particular analysis road inclination is set to zero) and rolling resistance can be determined, aggregated and utilised as total vehicle resistance force. Using (11), including rotating mass inertias for the engine and drivetrain, tractive effort can be calculated. Combining the tractive effort and vehicle resistance blocks, finally work at the wheels can be determined as net longitudinal force. A future article [18] will develop this longitudinal model into a rear-facing model to determine energy management strategies for motor usage and regeneration, as outlined in (24).

Equations (22) and (23) can be calculated simultaneously to determine lateral vehicle dynamics as detailed in the bicycle model. Combining the lateral and longitudinal models a vehicle model can be developed to simulate yaw plane motion. A X-Y table was produced to determine vehicle steering input versus time. The simulation duration and vehicle speed was determined by the NEDC, and steered wheel input (radians) was added during the braking and acceleration transitions to mimic slowing for a corner and accelerating away from the apex that is not normally considered in the NEDC. Control algorithms for vehicle stability can subsequently be determined by targeting neutral steer either through front and rear axle accelerations, corner radius versus steering angle or the differential of slip angle for front and rear tyres.

\section{CONCLUSIONS}

This article has developed and validated a Simulink ${ }^{\circledR}$ Hybrid Electric Vehicle dynamics model for the control of energy management and vehicle stability. The lateral model utilizing Pacejka's magic formula tyre model yields realistic responses and, with some tyre data manipulation, simulated outputs very closely match measured data. Under-steer and over-steer can be calculated and used as an input for stability control. With the inclusion of the longitudinal model, a predictive 2-D yaw plane (roll and pitch are ignored) model can be developed to include energy management strategies working in harmony with stability control. Lateral and longitudinal inputs can be predefined by the user to coincide with normal road driving or for that of a race circuit. The model is open loop allowing the user to create their own electrical hybrid system. The model will be used to develop stability and energy management strategies in an integrated longitudinal and lateral model, whether it be for road HEV's or Race Hybrids such as those used in Formula One and Endurance racing. 


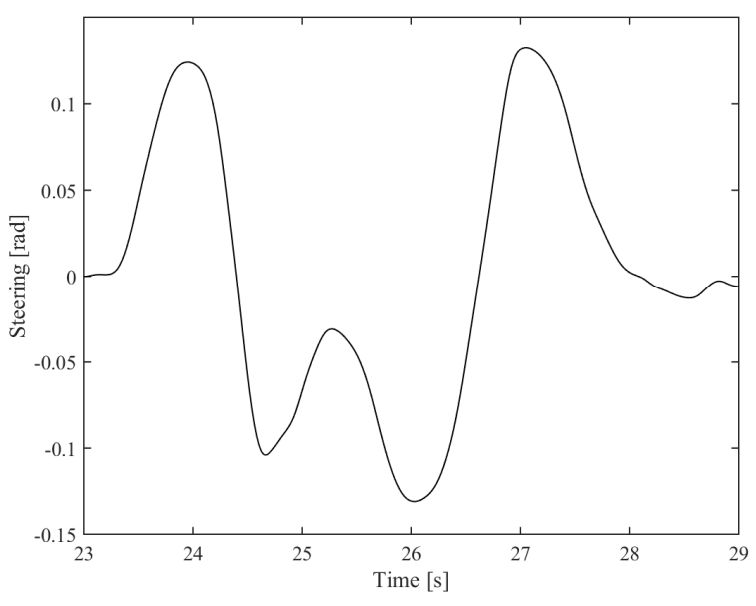

Figure 3. Steered wheel angle data $(38 \mathrm{~km} / \mathrm{h})$.

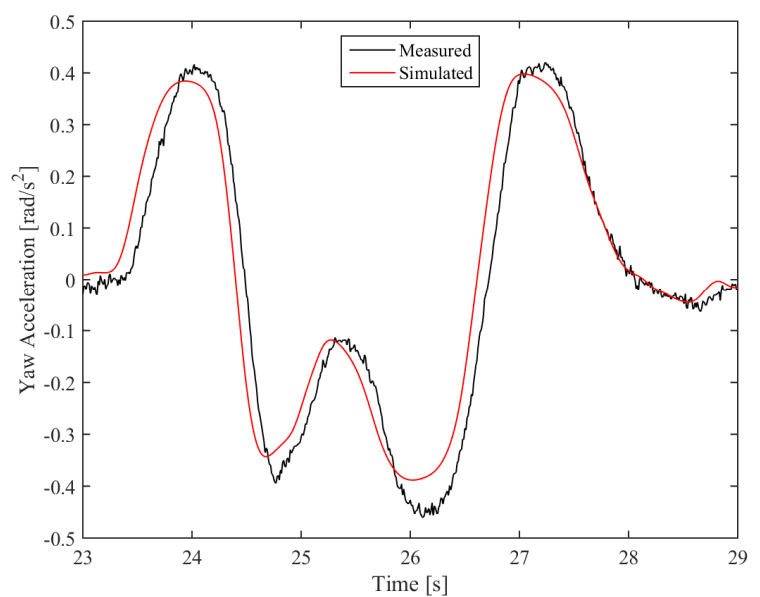

Figure 4. Yaw acceleration rate results, measured versus simulated.

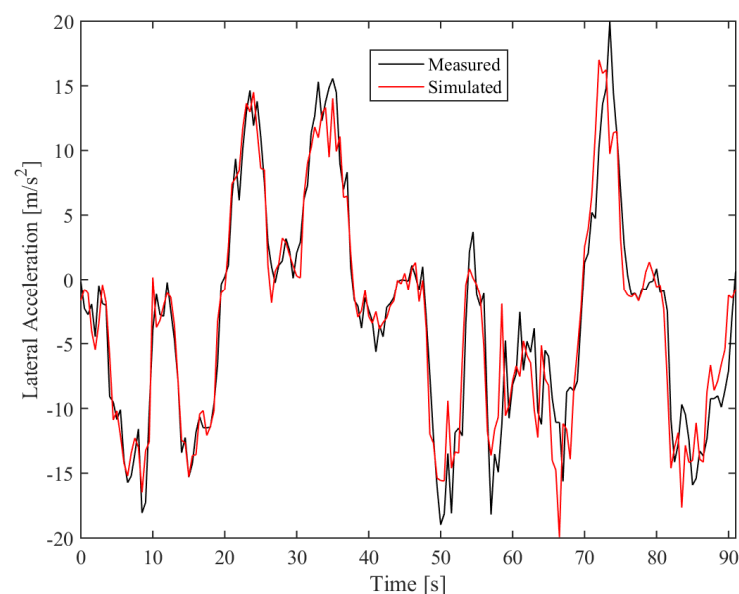

Figure 5. Lotus Evora Lateral Acceleration, measured versus simulated.

\section{ACKNOWLEDGMENTS}

Special thanks to Kristoffer Lundahl and his associates at Linköping University, Sweden, for their generosity in sharing measured data from their vehicle testing.

\section{REFERENCES}

[1] T. J. Barlow, S. Latham, I. S. McCrae and P. G. Boulter. "A reference book of driving cycles for use in the measurement of road vehicle emissions" Published Project Report, VERSION 3, 2009.

[2] GT-Suite Vehicle Driveline and HEV Application Manual V7.5, Gamma Technologies, 2015.

[3] Adams/Car User Manual, 2014.

[4] K. B. Wipke, M. R. Cuddy and S. D. Burch "ADVISOR 2.1: A userfriendly advanced powertrain simulation using a combined backward/forward approach" IEEE Transactions on Vehicular Technology, vol. 48, No. 6, pp. 1751-1761, Nov. 1999.

[5] S. Li and L. Hec "Co-simulation of Vehicle ESP System Based on ADAMS and MATLAB" Journal of Software, VOL 6, No. 5, pp. 866872, May 2011.

[6] D.W. Gao and A. Emadi "Modelling and Simulation of Electric and Hybrid Vehicles" Proceedings of the IEEE, vol. 95, No. 4, pp. 729745, April 2007.

[7] F. Yan, J. Wang and K. Huang "Hybrid Electric Vehicle Model Predictive Control Torque-Split Strategy Incorporating Engine Transient Characteristics" IEEE Transactions on Vehicular Technology, vol. 61, No. 6, pp. 2458-2467, July 2012.

[8] T. Hofman, M. Steinbuch, R. van Druten and A. Serrarens "Rulebased energy management strategies for hybrid vehicles" International Journal Electric and Hybrid Vehicles, vol. 1, No. 1, pp. 71-94, 2007.

[9] Volkswagen Audi Group. We have broken the most important part of ourvehicles:yourtrust.Available:http://www.volkswagen.co.uk/owner s/dieselinfo. Last accessed 2nd Nov. 2015.

[10] Environmental Protection Agency. EPA Conducted Confirmatory Tests. Available: http://www3.epa.gov/otaq/cert/documents/cd-mfrguid-1tr-2015-09-25.pdf. Last accessed 2nd Nov. 2015.

[11] H. B. Pacejka, Tyre and Vehicle Dynamics. 2nd ed. Oxford: Butterworth-Heinemann. pp. 61-64. 2006.

[12] M. Ehsani, Y. Gao and A. Emadi, Modern Electric, Hybrid Electric and Fuel Cell Vehicles. Florida: CRC Press. Ch. 2. 2009.

[13] R. Bosch, Bosch Automotive Handbook. 8th ed. Cambridge (MA): Bentley Publishers. 2014.

[14] E. Bakker, L. Nyborg and H. B. Pacejka "Tyre Modelling for use in Vehicle Dynamics Studies”. SAE Paper 870421. vol. 1, No. 1, pp. 1-15. 1987.

[15] J. R. Ellis, Vehicle Handling Dynamics. Hoboken: Wiley-Blackwell. 1994.

[16] K. Lundahl, J. Åslund and L. Nielsen (2013b). Vehicle Dynamics Platform, Experiments, and Modeling Aiming at Critical Maneuver Handling. Technical Report LiTH-R-3064. Department of Electrical Engineering, Linköping University, Sweden.

[17] ISO 3888-1 Double lane change test standards and procedures. 1999.

[18] K. Reeves, A. Montazeri and C.J. Taylor "Model Development and Energy Management Control for Hybrid Electric Race Vehicles". Submitted to UKACC International Control Conference, Belfast, August-September 2016 